\title{
Performances of a Steam Generation System by the Heat Utilization of
}

\section{Mesothermal Solar}

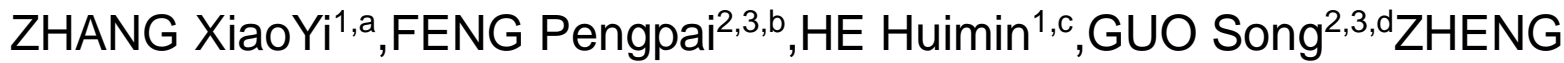

$$
\operatorname{Xin}^{2,3, e}
$$

(1. State Grid Jibei Energy Saving Service Co.,Ltd;Beijing 100045, China;

2. NARI Group Corporation (State Grid Electric Power Research Institute), Nanjing 210000, China;

3. NARI (Wuhan) Electrical Equipment \&Engineering Efficiency Evaluation Center, Wuhan 430074, China)

a478390697@qq.com,bfengpengpai@sgepri.sgcc.com.cn,1274491674@qq.com, guosong@sgepri.sgcc.com.cn, zhengxin1@sgepri.sgcc.com.cn

Keywords: solar thermal application; low-pressure steam; thermodynamic model; collecting temperature

Abstract: A 310kW steam generation system by the heat utilization of mesothermal solar was built and the thermodynamic model of main parts-solar collector and heat transformer were developed. In different conditions, the impact of solar radiation intensity, recycle ratio of condensate water and ambient temperature on thermal efficiency and heating power was investigated and the relationship of collecting temperature between system property was discussed. The result shows that the system property enhances significantly with the rise of solar radiation intensity. Recycling condensate water could escalate heating power while having little effect on thermal efficiency. The system property enhances first and then drops with the rise of ambient temperature. The system has the optimum collecting temperature which increases with the rise of solar radiation intensity and ambient temperature.

\section{Introduction}

As one of the important means to replace fossil energy and promote sustainable development, solar energy heat utilization technology is an effective way to realize energy saving and energy substitution $^{[1]}$. At present, solar heat utilization technology can be divided into three kinds according to the range of output temperature: low temperature technology $\left(<90^{\circ} \mathrm{C}\right)$ is mainly used in the production of domestic hot water and low temperature heating; high temperature utilization technology $\left(>130^{\circ} \mathrm{C}\right)$ mainly concentrates on the field of thermal power generation; medium temperature utilization $\left(90 \sim 130^{\circ} \mathrm{C}\right)$ is mainly used in the form of low pressure steam, which is widely used in the field of life and industry ${ }^{[2]}$.Absorption heat transformer (AHT) is a heat transfer device which can promote low-grade thermal energy to utilization.The solar collector combined with AHT is an effective heat utilization mode, which theoretically can obtain low pressure steam which is more than $100^{\circ} \mathrm{C}^{[3]}$.

At present, the integration of solar collector and AHT has been studied at home and abroad.Gommed demonstrated theoretically the possibility of producing industrial steam using solar energy AHT system ${ }^{[4,5]}$. Jiang Junzhi and Pei Gang studied the medium temperature steam 
generation system which was combined by parabolic solar collector and AHT, analyzed the heat transfer characteristics and transmission power, obtained comprehensive evaluation of derived heat transfer enhancement criterion ${ }^{[6,7]}$. Gormi and Liu Xiaohua designed a device that included flat plate solar collector and AHT to desalinate sea water. Based on this, the operating characteristics of each part of the system were modeled and analyzed under different operating conditions ${ }^{[8,9]}$.Tao Yubing and He Zhibing established model for heat transfer process of the collector and heat conversion in the system. Energy flux density distribution were studied by numerical simulation and the heat loss was measured ${ }^{[10,11]}$.

In this paper, a set of medium temperature steam system of solar energy heat utilization was established. The system was composed of solar collector array and AHT. The thermodynamic models were established respectively and the energy balance equations of all the system parts were given. Based on these, the thermodynamic analysis of the system was carried out, the influence on the system performance from environmental parameters and process parameters under the design conditions and variable conditions was studied.

\section{System model}

The medium temperature solar heating system is mainly composed of two modules, solar collector array and thermal converter (AHT). The system principle is shown in figure 1.

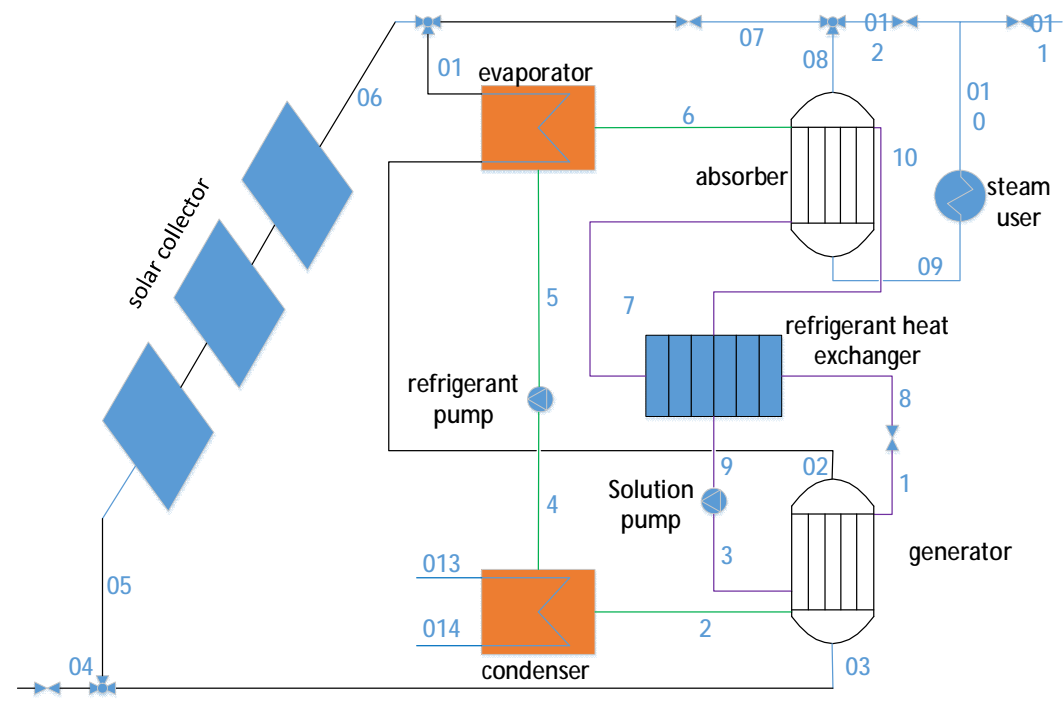

Fig.1 Principle diagram of the system

In the process of generating steam, feedwater (04) converting to steam (09) undergones two endothermic processes. In the first stage, feedwater (04) is preheated in solar collector array and is heated to hot water (06) whose temperature has been significantly improved. Part of the hot water (01) which flows past the flow valve is used as driving source of AHT system.In the second stage, the other part of the hot water (07) and condensed water (012) are mixed into the heated water (08), which absorbs heat and tures into low pressure steam (09) for users.

AHT cycle principle: hot water (01) works as heat source, which returns to the solar collector again after flowing through the evaporator and generator to complete to heat cycle. $\mathrm{LiBr}-\mathrm{H}_{2} \mathrm{O}$ is chosed as AHT refrigerant. After exchanging heat with hot water (012) in the AHT, $\mathrm{LiBr}-\mathrm{H}_{2} \mathrm{O}$ dilute solution becomes concentrated solution and refrigerant steam (2) is generated. The refrigerant steam (2) is condensed into saturated refrigerant water (4), then flowes into high-pressure evaporator through cooling pump. Saturated refrigerant water (4) driven by hot water (01) tures into high pressure refrigerant steam (6) in the high-pressure evaporator. High pressure refrigerant steam (6) is mixed with $\mathrm{LiBr}-\mathrm{H}_{2} \mathrm{O}$ concentrated solution. In the above progress, a lot of heat is absorbed by hot 
water (08). The mixed $\mathrm{LiBr}-\mathrm{H}_{2} \mathrm{O}$ dilute solution(7) flows back to cycle.

\section{Solar collector model}

In order to establish the mathematical model of the solar collector, the following assumptions are made.

a. The solar radiation intensity and environmental temperature are constant;

b. The solar collector is studied as a whole, dust influence on collector efficiency is ignored;

c. The process of thermal cycle is stabe, without considering the heat loss of pipeline.

When the collector runs stably, the relationships of system effective heat collection $Q_{e}$, total acceptance of solar radiation $Q_{s}$ and heat collector efficiency are shown blow:

$$
\begin{gathered}
Q_{e}=Q_{s} \cdot \eta_{c} \\
Q_{e}=m_{05} C_{p}\left(T_{06}-T_{05}\right) \\
Q_{s}=A_{c} \cdot I_{s} \\
\eta_{c}=\alpha_{0}-\alpha_{1} \frac{T_{05}-T_{a}}{I_{s}}
\end{gathered}
$$

Where:

$m_{05}$ :is the flow rate of the working fluid (water) of a collector $(\mathrm{kg} / \mathrm{s}) ; \quad C_{p}=4.18 \mathrm{~kJ} /\left(\mathrm{kg} \cdot{ }^{\circ} \mathrm{C}\right)$, is specific heat capacity of water at constant pressure; $T_{06}$ is heat collection temperature (i. e. collector temperature $\left({ }^{\circ} \mathrm{C}\right) ; T_{05}$ :is inlet temperature of thermodynamic mass $\left({ }^{\circ} \mathrm{C}\right) ; A_{c}$ :is effective area of plate heat collector $\left(\mathrm{m}^{2}\right) ; \quad I_{s}$ :is solar radiation intensity $\left(\mathrm{kW} / \mathrm{m}^{2}\right) ; T_{a}$ :is ambient temperature $\left({ }^{\circ} \mathrm{C}\right) ; \alpha_{0}:$ is collector truncation efficiency $(\%) ; \quad \alpha_{1}$ : is first correction factor of collector $\left(\mathrm{kW} /\left(\mathrm{m}^{2} \cdot{ }^{\circ} \mathrm{C}\right)\right)$.

\section{Absorption heat transformer model}

In order to establish the mathematical model of heat converter, the following assumptions are made:

a. The heat transfer process is stable, without considering the heat loss and the flow resistance;

b. The refrigerant solution of generator and evaporator outlet are saturated, so as the refrigerant vapor of absorber and condenser outlet;

c. The differential pressure of refrigerant pump and ignore solution pump pressure is neglected.the enthalpy value remains unchanged before and after throttling.

The mathematical model of heat converter includs mass conservation equation and energy conservation equation of refrigerants.

The heat balance equations of converter components are shown in table 1 . 
Tab.1 Balance equation of heat transformer

\begin{tabular}{|c|c|}
\hline Parts & Equations \\
\hline Evaporator & $\begin{array}{l}Q_{f f}=m_{6} h_{6}-m_{5} h_{5} \\
Q_{f f}=m_{01} h_{01}-m_{02} h_{02}\end{array}$ \\
\hline Generator & $\begin{array}{c}Q_{f s}=m_{3} h_{3}+m_{2} h_{2}-m_{1} h_{1} \\
Q_{f s}=m_{02} h_{02}-m_{03} h_{03}\end{array}$ \\
\hline Absorber & $\begin{array}{c}Q_{x s}=m_{6} h_{6}+m_{10} h_{10}-m_{7} h_{7} \\
Q_{x s}=m_{09} h_{09}-m_{08} h_{08}\end{array}$ \\
\hline Condenser & $\begin{array}{c}Q_{\mathrm{ln}}=m_{2} h_{2}-m_{4} h_{4} \\
Q_{\mathrm{ln}}=m_{014} h_{014}-m_{013} h_{013}\end{array}$ \\
\hline Heat exchanger & $\begin{array}{l}Q_{h r}=m_{10} h_{10}-m_{9} h_{9} \\
Q_{h r}=m_{7} h_{7}-m_{8} h_{8}\end{array}$ \\
\hline Solution pump & $W_{P_{1}}=m_{3}\left(P_{9}-P_{3}\right) / \eta_{p}$ \\
\hline Refrigerant pump & $W_{P 2}=m_{4}\left(P_{5}-P_{4}\right) / \eta_{p}$ \\
\hline
\end{tabular}

The heat converter efficiency is expressed as follows in stable operation condition:

$$
\eta_{A H T}=\frac{Q_{x s}}{Q_{z f}+Q_{f s}}
$$

The condensate recovery ratio $\varepsilon$ is expressed as the percentage of the total amount of condensate used in the recovery process, which is calculated as follows in this system:

$$
\varepsilon=m_{012} / m_{010} \times 100 \%
$$

The external heating power of the system:

$$
Q_{o}=m_{09}\left(h_{09}-h_{010}\right)
$$

System thermal efficiency:

$$
\eta=\frac{Q_{o}}{Q_{s}}
$$

\section{System running state and performance analysis}

\section{Performance parameters in design conditions}

On the basis of the established system thermodynamics model and the assumption condensate recovery ratio 0 , the indexes of the system performance parameters were calculated which are shown in table 2 according to the temperature difference of each component. When the refrigerant heat grade is improved, the heat transfer efficiency is reduced, so it directly affects the thermal efficiency of the whole transport system. 
Tab.2 Performances under designed condition

\begin{tabular}{cc}
\hline Parameters & Values \\
\hline Radiation intensity $\mathrm{I}_{\mathrm{s}} /\left(\mathrm{W} \cdot \mathrm{m}^{2}\right)$ & 800 \\
Ambient temperature $\mathrm{T}_{\mathrm{a}} /{ }^{\circ} \mathrm{C}$ & 25 \\
Collector temperature $\mathrm{T}_{06} /{ }^{\circ} \mathrm{C}$ & 85 \\
Reclaimed water temperature $\mathrm{T}_{03} /{ }^{\circ} \mathrm{C}$ & 75 \\
Low pressure steam flow $\mathrm{m}_{09} /\left(\mathrm{kg} \cdot \mathrm{s}^{-1}\right)$ & 0.278 \\
Evaporator heat $\mathrm{Q}_{\mathrm{z}} / \mathrm{kW}$ & 352 \\
Generator heat $\mathrm{Q}_{\mathrm{fs}} / \mathrm{kW}$ & 323 \\
Absorber heat $\mathrm{Q}_{\mathrm{xs}} / \mathrm{kW}$ & 324 \\
Condenser heat $\mathrm{Q}_{\mathrm{ln}} / \mathrm{kW}$ & 704 \\
Solar radiation $\mathrm{Q}_{\mathrm{s}} / \mathrm{kW}$ & 1114 \\
Heating power $\mathrm{Q}_{\mathrm{o}} / \mathrm{kW}$ & 310 \\
Collector efficiency $\eta_{\mathrm{c}}$ & $63.9 \%$ \\
Heat converter efficiency $\eta_{\mathrm{AHT}}$ & $47.9 \%$ \\
System thermal efficiency $\eta$ & $27.8 \%$ \\
\hline
\end{tabular}

\section{System performance analysis under variable operating conditions}

The influence on system performance from operation parameters including radiation intensity $I_{s}$, condensate recovery ratio $\varepsilon$ and ambient temperature $T_{a}$ were studied. The import flow remained unchanged.

Figure 2 shows the influence on system performance of radiation intensity with the recycled water recovery ratio $\varepsilon$ and the ambient temperature $T_{a}$ being design value when the recycled water $T_{03}$ is $75^{\circ} \mathrm{C}$. When $I_{s}$ changes from $0.2 \mathrm{~kW} / \mathrm{m}^{2}$ to $1.0 \mathrm{~kW} / \mathrm{m}^{2}$, the collector efficiency increases from $28 \%$ to $71.2 \%$, the system heat accumulation effectively increases. When the heat collecting temperature $T_{06}$ increases, thermal converter circulating temperature is improved, circulation rate decreases, $\eta_{\text {AHT }}$ increases about $3 \%$. The heating power of the whole system $Q_{o}$ increases by 11.8 times and the overall thermal efficiency of the system increases by $20 \%$. It can be seen that the increase of radiation intensity has significantly improved the performance of the system.

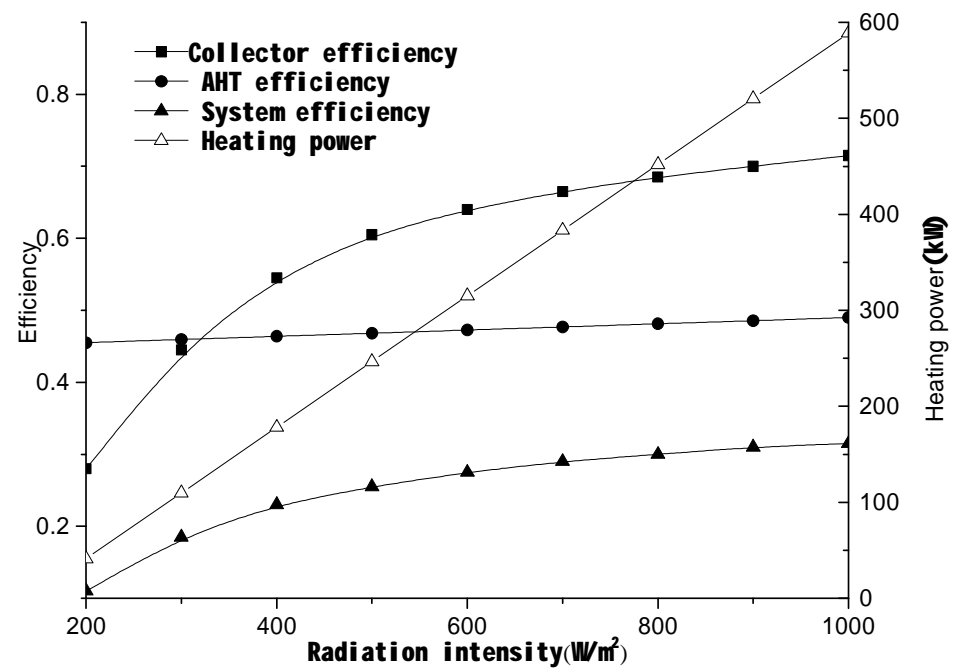

Fig.2 The rule of property changing with collecting temperature

Figure 2 shows the influence of the condensate recovery ratio $\varepsilon$ on the system performance when the radiation intensity and the ambient temperature are at designed values. It can be obtained 
from the diagram that $\varepsilon$ has little influence on $\eta_{c}$ and $\eta_{A H T}$.For the system, the condensate temperature is higher. When the condensate recovery flow increases, the collector heating is reduced, the thermal efficiency of the system is increased by $2 \%$ and the heating power is raised by $30 \mathrm{~kW}$.

In summary, recovery of condensate has little effect on the efficiency, but the influence on heating power is more obvious. In the condition of condensate water being recycled for heating occasions, increasing the pipeline equipment investment can get a good heating capacity upgrade.

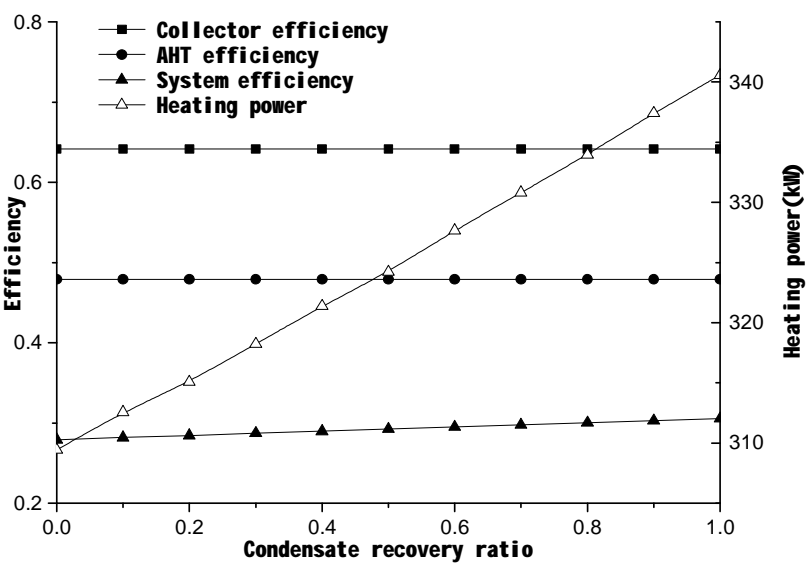

Fig. 3 The rule of property changing with recycle ratio of condensate water

Figure 3 shows the influence of ambient temperature $T_{a}$ on the system performance when the radiation intensity $I_{s}$ and condensate recovery ratio $\varepsilon$ are at designed values.As the diagram shows, with the increase of the environmental temperature, the feedwater temperature and the input heat of the system increase, and heat loss of heat collector is reduced, and $\eta_{c}$ is improved by $6.5 \%$. For AHT, with working temperature of condenser heat rising, circulation rate increases, $\eta_{A H T}$ decreases.But when the temperature is above $30^{\circ} \mathrm{C}$, pressure difference of evaporation/occurrence process increases, there is a sudden drop in the saturation concentration of the refrigerant solution. The absorption capacity of concentrated solution decreases obviously, System heat transfer performance deteriorates, $\eta_{A H T}$ decreases rapidly.Being affaected by AHT performance, the system thermal efficiency $\eta$ and heating power $Q_{o}$ first slowly rise and then decline rapidly in the experimental temperature range. Extreme point is obtained at $25^{\circ} \mathrm{C}$.

The above analysis is based on the fact that the feedwater flow and heat source water are constant. When $T_{a}$ is above $30^{\circ} \mathrm{C}$, working temperatures of heat converter components are regulated by changing the heating water flow. Thus pressure difference of evaporation/occurrence process decreases, $\eta_{A H T}$ changes moothly, system performance tends to be stable. 


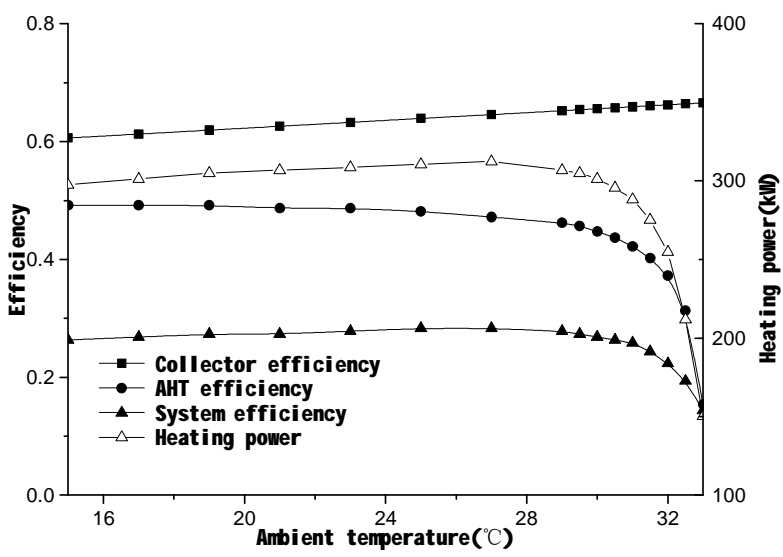

Fig.4 The rule of property changing with ambient temperature

\section{Influence of collector temperature on system performance}

Under design conditions, when the collector temperature $T_{06}$ rises from $82^{\circ} \mathrm{C}$ to $98^{\circ} \mathrm{C}$, the performance of the system is shown in figure 5. With the rise of $T_{06}$, collector efficiency $\eta_{c}$ declines, AHT efficiency $\eta_{A H T}$ first increases rapidly then becomes stable.Affected by this, system thermal efficiency $\eta$ and heating power $Q_{o}$ first increase rapidly and then decrease slowly, and the maximum value is about $88^{\circ} \mathrm{C}$.Thus, there is optimum of the system performance with the collector temperature changing. It is called the optimum collector temperature $T_{M}$ which is of great significance. The further analysis of the optimum collector temperature under different environmental conditions is made.

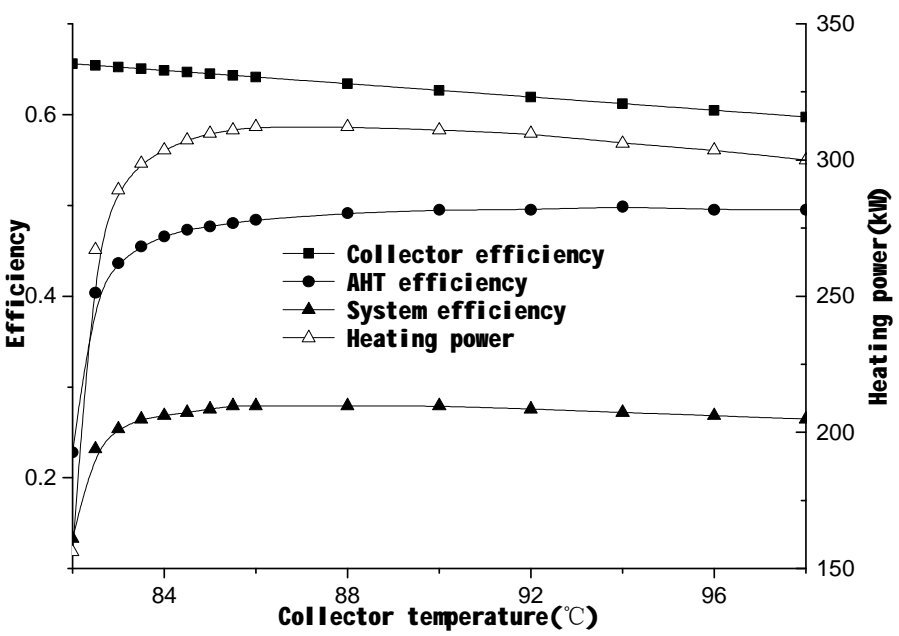

Fig.5 The rule of property changing with collecting temperature

Figure 6 shows the variation of the system thermal efficiency $\eta$ and the heating power $Q_{o}$ with the variation of collector temperature at different values of the radiation intensity $I_{s}$. When $I_{s}$ increases from $0.3 \mathrm{~kW} / \mathrm{m}^{2}$ to $0.9 \mathrm{~kW} / \mathrm{m}^{2}, T_{M}$ rises from $79 \mathrm{C}$ to $94 \mathrm{C}, \eta_{\max }$ increases from $18.9 \%$ to 
$31 \%, Q_{o \max }$ increases from $107 \mathrm{~kW}$ to $518 \mathrm{~kW}$. And before $T_{06}$ arrives at $T_{M}$, the system performance improves faster than exceeding $T_{M}$.It can be seen that in the given range, as $I_{s}$ increases, the corresponding maximum collector temperature increases continuously with the maximum value of the heat increases accordingly.

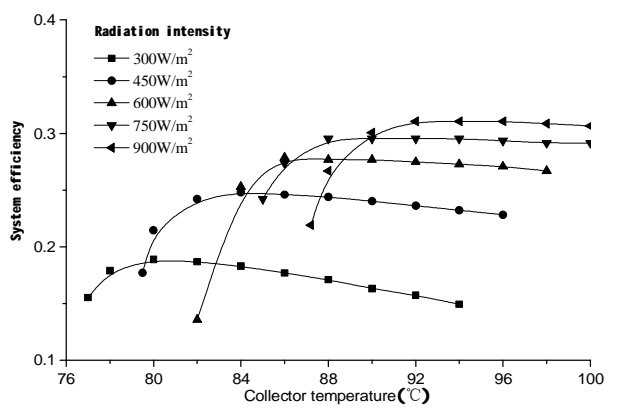

(a) System efficiency

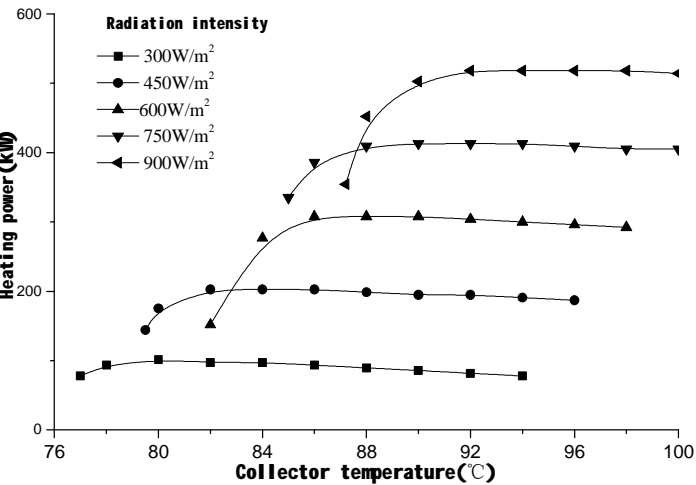

(b) Heating power

Fig.6 The rule between property and collecting temperature under different radiation intensity

Figure 7 shows the variation of system heat efficiency $\eta$ and the heating power $Q_{o}$ with the variation of collector temperature when the condensate recovery ratio is at different value.In the process of $\varepsilon$ changing from 0 to $1, T_{M}$ maintains at 88 degrees, $\eta_{\max }$ increases from $28 \%$ to $30.6 \%, Q_{o \max }$ increases from $311 \mathrm{~kW}$ to $341 \mathrm{~kW}$. Thus, the influence of the condensate recovery ratio $\varepsilon$ on the optimum collector temperature $T_{M}$ is negligible.

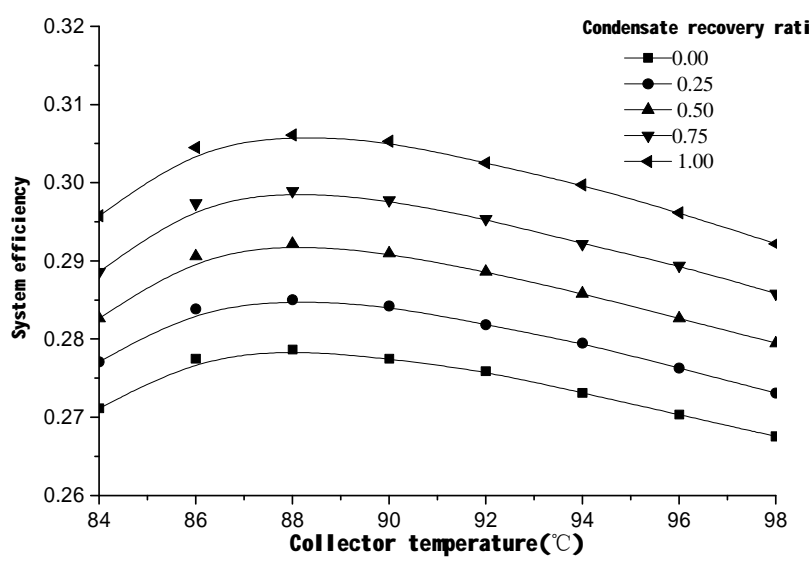

(a) System efficiency

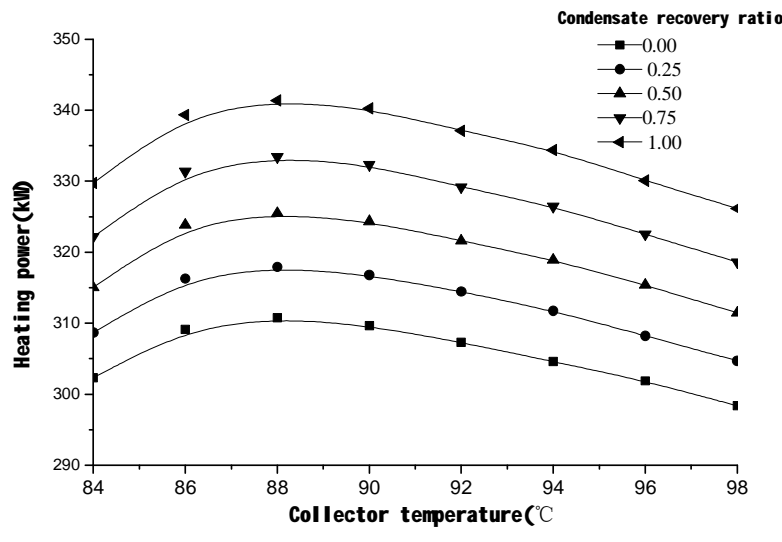

(b) Heating power

Fig.7 The rule between property and collecting temperature under different recycle ratio of condensate water

Figure 8 reflects the ambient temperature $T_{a}$ at different values, the system thermal efficiency $\eta$ and heating power $Q_{o}$ with the collector temperature changing. In the process of $T_{a}$ changing from $15^{\circ} \mathrm{C}$ to $35^{\circ} \mathrm{C}, T_{M}$ increases from $80^{\circ} \mathrm{C}$ to $92^{\circ} \mathrm{C}$, the corresponding $\eta_{\max }$ increases from $26.7 \%$ to $29.2 \%, Q_{o \max }$ increases from $300 \mathrm{~kW}$ to $324 \mathrm{~kW}$. It can be seen that within the given range, 
with the increase of $T_{a}$, the corresponding optimal collector temperature $T_{M}$ is increasing, and the maximum value of $\eta$ and $Q_{o}$ rises accordingly.

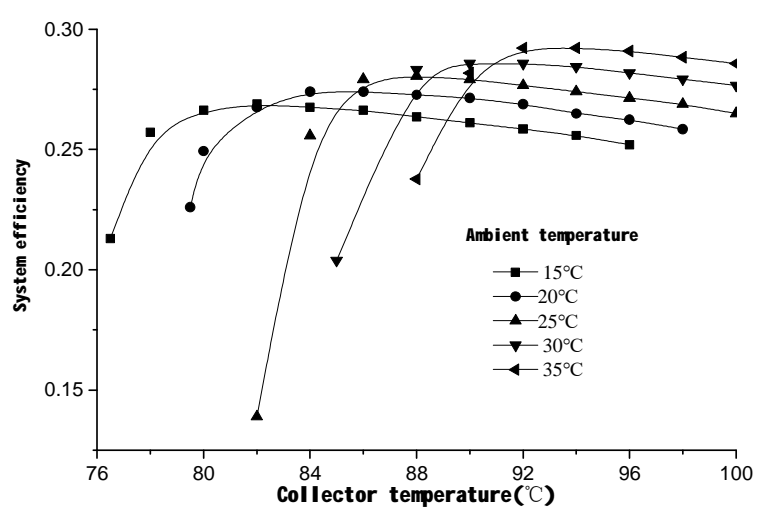

(a) System efficiency

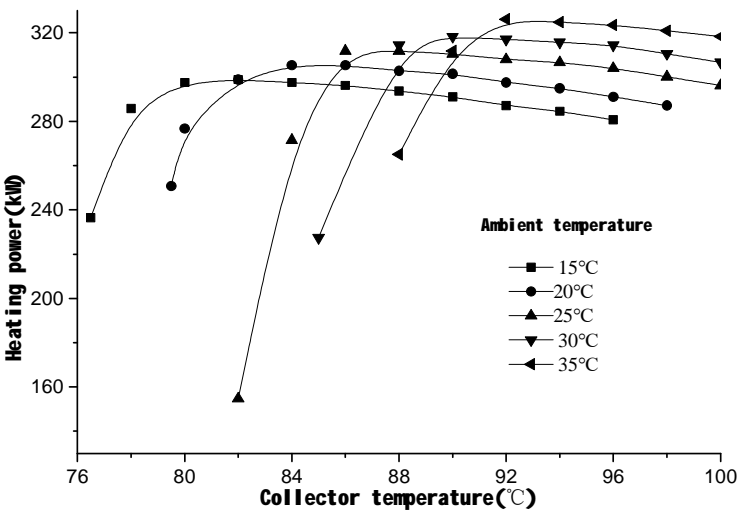

(b) Heating power

Fig. 8 The rule between property and collecting temperature under different ambient temperature

\section{Conclusion}

In this paper, a thermodynamic model of the steam system for the solar heat change was established, the performance of the system under the design conditions and off-design conditions was analyzed. By studying the influence of solar radiation intensity, environmental temperature, condensate recovery ratio and collector temperature on system efficiency and heating power, the following conclusions are obtained.

(1) Increasing the solar radiation intensity has a significant improvement on the system performance. Under the design condition, when the radiation intensity increases from $0.2 \mathrm{~kW} / \mathrm{m}^{2}$ to $1.0 \mathrm{~kW} / \mathrm{m}^{2}$, the efficiency of the system increases by $20 \%$, and the heating power increases by 11.8 times.

(2) The recovery of condensate has little effect on the efficiency, but the improvement of heating power is more obvious. Under the design condition, the system efficiency is increased by $2 \%$, and the heating power is raised by $30 \mathrm{~kW}$ when the condensate recovery ratio changes from 0 to 1. In the condition of condensate water being recycled for heating occasions, increasing the pipeline equipment investment can get a good heating capacity upgrade.

(3) In a certain temperature range, the increase of ambient temperature is beneficial to improve system efficiency and heating power. When the ambient temperature exceeds a certain value (design conditions for $30^{\circ} \mathrm{C}$ ), performance of heat exchanger, system efficiency and heating power are drastically reduced. At this point, the performance of the system can be improved by changing the heat source water flow to adjust the working temperature of the heat change device.

(4) With the increase of collector temperature, the system efficiency and heating power increase rapidly to the maximum and then decline gradually. The optimum collector temperature is independent of the condensate recovery ratio, which increases with the increase of radiation intensity and ambient temperature. When operating under different conditions, the collector temperature should be optimized as much as possible so that the system efficiency and heating capacity can be optimized. 


\section{Reference:}

[1] ZHAO Jun, GAO Liu-hua. Solar thermal utilization technology research progress and countermeasures[J]. Construction Science and Technology, 2012, 21:34-37.

[2] CHEN Xiu-juan, LIU Zhen-hua. Experimental research of medium temperature open heat pipe solar collector[J]. Journal of Thermal Science and Technology, 2012, 03: 272-276.

[3] Ma Guang-bai, ,Ni Chao, Zhou Ling. Preliminary experiments of thermal performance of solar collector for medium temperature applications[J]. Acta Energiae Solaris Sinica, 2010, 05: 588-591.

[4] Gommed K, Grossman G. Process steam generation by temperature boosting of heat from solar ponds[J]. Solar Energy, 1988, 41(1): 81-89.

[5] Sown A. Effect of irreversibilities on performance of an absorption heat transformer used to increase solar pond's temperature[J]. Renewable Energy , 2003, 29(4): 501-515.

[6] JIANG Jun-zhi. Research on The Heat Transfer of Flooded-type Solar Steam Generator and Structural Design[D]. Huazhong University of Science and Technology, 2011.

[7]PEI Gang, YANG Jin-wei, ZHANG Tao, et al. Research of flat heat pipe solar collector device[J]. Journal of Thermal Science and Technology, 2011, 02: 138-141.

[8]Gomri R. Energy and exergy analyses of seawater desalination system integrated in a solar heat transformer[J]. Desalination, 2009, 249(1): 188-196.

[9]LIU Xiao-hua, ZHANG Xin-chun, SHEN Sheng-qiang, ZHU Ming-ming. Performance analysis on fluctuation-load condition of low temperature multi-effect solar distillation system with evacuated tube collectors[J]. Journal of Thermal Science and Technology, 2015, 02: 149-154.

[10]TAO Yu-Bing, HE Ya-Ling, LIU Ying-Wen. Numerical study on coupled heat-transfer process in a parabolic solar collector[J]. Journal of Engineering Thermophysics, 2009, 09: 1543-1545.

[11]HE Zhi-bing, LIU Zhen-hua, ZHAO Feng, XIAO Hong-seng. Design and experimental study of simplified CPC style medium and high temperature evacuated tubular solar collectors with concentric copper tube.[J]. Journal of Thermal Science and Technology, 2013, 01: 90-94. 